\title{
Processamento e avaliação das propriedades de tubos cerâmicos porosos para microfiltração de emulsões
}

\author{
(Processing and evaluation of porous ceramics for micro \\ filtration of emulsions)
}

\author{
D. S. Rosa, V. R. Salvini, V. C. Pandolfelli \\ Departamento de Engenharia de Materiais - DEMa \\ Universidade Federal de S. Carlos - UFSCar \\ Rod. Washington Luiz, km 235, C.P. 676, S. Carlos, SP 13565-905 \\ darosa_99@uol.com.br,vicpando@power.ufscar.br
}

\begin{abstract}
Resumo
Cerâmicas porosas têm sido utilizadas em diversas aplicações tecnológicas como coletores de partículas sólidas, isolantes térmicos e acústicos. As áreas de biotecnologia e biomedicina utilizam cerâmicas porosas como reatores em processos de fermentação e em implantes, respectivamente. Adicionalmente, estes materiais apresentam grande aplicação na área de microfiltração de líquidos e emulsões, como a purificação de combustíveis através da separação da água e óleo, e na separação das proteínas do leite. O objetivo deste trabalho é o de produzir tubos de cerâmicas porosas para microfiltração de combustíveis. A primeira etapa compreendeu a produção de tubos cerâmicos porosos por colagem de suspensões e avaliação de suas propriedades físicas, fluidodinâmicas e mecânicas finais. Já a segunda etapa consistiu na análise do desempenho destes tubos durante a microfiltração de combustíveis. As propriedades finais das cerâmicas porosas foram avaliadas em função das variações na composição da suspensão e nas condições de queima. Após queima, os tubos cerâmicos apresentaram porosidade na faixa de 48 a $65 \%$, distribuição estreita de diâmetro de poros menores que $1 \mu \mathrm{m}$, e constantes de permeabilidade Darciana $\left(\mathrm{k}_{1}\right)$ e não-Darciana $\left(\mathrm{k}_{2}\right)$ da ordem de $10^{-15} \mathrm{~m}^{2}$ e $10^{-11} \mathrm{~m}$, respectivamente. Essas propriedades são similares aos tubos comerciais importados e, portanto, essas cerâmicas porosas desenvolvidas têm um grande potencial de aplicação em processos de microfiltração.
\end{abstract}

Palavras-chave: alumina porosa, processamento cerâmico, microfiltração.

\begin{abstract}
Porous ceramics have been applied in a broad range of technological purposes, such as dust collectors, thermo-acoustic insulators and burners. In the biotechnology and biomedicine areas, porous ceramics have been applied as reactors in fermentation processes and as implants, respectively. Besides that, they can also be used in the micro filtration process of liquids and emulsions, such as fuel purification through separation of water from oil, and in the separation of proteins of the milk. The objective of this work is the production of porous ceramics tubes for fuel micro filtration. The first stage of the research was the production of porous ceramics by colloidal processing and evaluation of its final physical, fluid dynamic and mechanical properties. In a second stage the performance of these ceramic tubes during the micro filtration of fuel was analysed. The final properties of porous ceramics were evaluated according to the changes in the composition and sintering conditions. After firing, the ceramic tubes had their porosity in the range of 48 to $65 \%$-vol, narrow distribution with pore diameters bellow than $1 \mu \mathrm{m}$ and constants of Darcian ( $\left.k_{\nu}\right)$ and non-Darcian $\left(k_{2}\right)$ permeability of $10^{-15} \mathrm{~m}^{2}$ and $10^{-11} \mathrm{~m}$, respectively. These properties are similar to the commercial imported products and therefore these ceramics have a great potential of application in micro filtration processes.
\end{abstract}

Keywords: porous alumina, ceramic processing, micro filtration.

\section{INTRODUÇÃO}

A microfiltração é um processo que usa uma membrana microporosa como barreira permeável na separação de duas fases [1]. Esse processo é aplicado no processamento de rejeitos industriais, indústria de alimentos, cosméticos, processos químicos entre outros [1-3]. Dentre os materiais para produção de membranas microporosas destacam-se os cerâmicos, devido à sua estabilidade química, dimensional, alta durabilidade e resistência a alta temperatura e pressão [2].

Comercialmente, os tubos cerâmicos porosos para microfiltração são formados por duas partes: um substrato e uma membrana, ambos porosos, mas com finalidades distintas. O substrato proporciona resistência mecânica em uso, apresenta porosidade na faixa de 30 a $40 \%$, poros de 1 a $3 \mu \mathrm{m}$ de diâmetro e espessura de parede em torno de $2 \mathrm{~mm}$ [24]. Já a membrana é depositada sobre a parede interna do 
tubo (substrato) e deve apresentar poros na faixa de 0,1 a $0,8 \mu \mathrm{m}[2,3]$. Este conjunto - substrato e membrana - deve resultar em uma constante de permeabilidade da ordem de $10^{-16} \mathrm{~m}^{2}$. Ainda segundo a literatura, para uma alta eficiência durante a microfiltração, os valores de fluxo devem estar na faixa de 15 a 90 ( $1 / \mathrm{m}^{2} \mathrm{~h}$ bar $)$ e o coeficiente de rejeição deve ser maior que $99 \%$ [2]. Este coeficiente indica a capacidade do filtro na redução do teor de impurezas da emulsão.

As membranas porosas podem ser fabricadas por diversas técnicas, tais como o "sol-gel" [2] e a colagem de barbotina [5]. Já o substrato (tubo poroso) pode ser produzido por colagem de suspensões com alta concentração de sólidos ou por extrusão [4]. Além da escolha da técnica de conformação destas partes é preciso, também, selecionar aditivos para a geração de poros. O mais comum é o uso de substâncias que durante a queima se decompõem e deixam em seu lugar poros, tais como os polímeros orgânicos $[1,2,4]$.

$\mathrm{O}$ uso de substâncias inorgânicas que sofrem transformação de fase acompanhada de redução volumétrica durante a queima também pode ser adotado para a fabricação de cerâmicas porosas $[2,7]$.

Assim, o objetivo do presente trabalho é o de produzir pelo método de colagem de suspensões e uso de aditivos inorgânicos tubos cerâmicos porosos para microfiltração de emulsões, tendo como diferencial a associação da membrana e do substrato em uma única camada.

\section{MATERIAIS E MÉTODOS}

A matéria-prima cerâmica utilizada foi a alumina calcinada (A1000 SG, Alcoa, Brasil) com densidade de $3,98 \mathrm{~g} / \mathrm{cm}^{3}$ e área superficial de $9 \mathrm{~m}^{2} / \mathrm{g}$. O defloculante escolhido foi o acido cítrico (Sigma) no teor de $0,31 \mathrm{mg} / \mathrm{m}^{2}$. Para a formação de poros substituiu-se $60 \%$-v da alumina por um aditivo inorgânico [2, 7]. Informações adicionais sobre o aditivo utilizado e sua síntese estão restritas, uma vez que se encontra em processo de patenteamento.

Para a primeira etapa do trabalho, que consistiu na avaliação das propriedades físicas e fluidodinâmicas das cerâmicas variando-se a fração de sólidos, tempo e temperatura de patamar de queima, corpos cerâmicos na

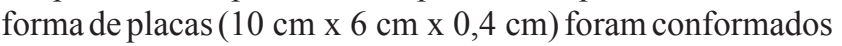
por colagem de suspensões. Para a segunda etapa, que teve como objetivo avaliar a eficiência de filtração do material cerâmico sob mesmas condições dos comerciais foram colados tubos com $25 \mathrm{~cm}$ de comprimento, $1 \mathrm{~cm}$ de diâmetro externo e $0,2 \mathrm{~cm}$ de espessura de parede.

Anterior a colagem das placas e tubos, a suspensão foi inicialmente homogeneizada em moinho de bolas por $1 \mathrm{~h} \mathrm{e}$, posteriormente, submetida a uma câmara de vácuo por $5 \mathrm{~min}$ para desaeração. Após $12 \mathrm{~h}$ as placas foram retiradas do molde de gesso, submetidas a secagem por $12 \mathrm{~h}$ a $50^{\circ} \mathrm{C}$, e posteriormente por mais $12 \mathrm{~h} \mathrm{a} 110^{\circ} \mathrm{C}$. Os tubos após $20 \mathrm{~min}$ da colagem, ainda no molde, foram submetidos a secagem por $12 \mathrm{~h}$ a $50^{\circ} \mathrm{C}$, e em seguida secos fora do molde a $110^{\circ} \mathrm{C}$ por mais $12 \mathrm{~h}$.
As placas cerâmicas foram sinterizadas a $1150^{\circ} \mathrm{C}$ e à $1250{ }^{\circ} \mathrm{C}[6]$ em dois tempos de patamares distintos ( 1 e $3 \mathrm{~h}$ ), com taxa de aquecimento de $5{ }^{\circ} \mathrm{C} / \mathrm{min}$.

A caracterização física das placas compreendeu a determinação de porosidade e densidade pelo método de Archimedes, permeabilidade ao ar à temperatura ambiente (PBT) e porosimetria de mercúrio. Das medidas de permeabilidade ao ar foram calculadas as constantes de permeabilidade $\mathrm{k}_{1}$ e $\mathrm{k}_{2}$, segundo a equação de Forchheimer:

$$
\frac{\mathrm{P}_{\mathrm{i}}^{2}-\mathrm{P}_{0}^{2}}{2 \mathrm{P}_{0} \mathrm{~L}}=\frac{\mu}{\mathrm{k}_{1}} \mathrm{v}_{\mathrm{s}}+\frac{\rho}{\mathrm{k}_{2}} \mathrm{v}_{\mathrm{s}}^{2}
$$

onde $\mathrm{P}_{\mathrm{i}}$ e $\mathrm{P}_{0}$ representam as pressões de entrada e saída do ar em Pascal $(\mathrm{Pa})$, respectivamente; $\mathrm{L}$ a espessura da amostra em metros $(\mathrm{m}) ; \mathrm{v}_{\mathrm{s}}$, a velocidade de escoamento em (m/s); $\mu$, a viscosidade em (Pa.s) e, $\rho$, a densidade do ar em $\left(\mathrm{Kg} / \mathrm{m}^{3}\right)$.

A variação da fração de sólidos, temperatura e tempo de sinterização geraram variações de porosidade que puderam ser observadas nos dados obtidos dos ensaios de imersão, de permeabilidade ao ar e de porosimetria de mercúrio. A partir da análise conjunta destes dados, selecionou-se a composição mais adequada à produção dos tubos para microfiltração.

Para os ensaios de microfiltração tangencial foi usada água pura como referência e duas emulsões, sendo uma de água com óleo de girassol ( $2 \%$-v) e outra acrescida de goma xantana $(1 \mathrm{~g} / \mathrm{L})$. Uma região do equipamento utilizado está ilustrado na Fig. 1.

A faixa de pressão durante os testes de microfiltração variou entre 0,5 e 6,5 bar. A vazão mássica de emulsão no sistema foi controlada pela freqüência de operação da bomba que foi de 25, 35, 45 e $55 \mathrm{~Hz}$. A pressão foi aumentada em 1 bar a cada 15 min e amostras de filtrado foram coletadas a cada $5 \mathrm{~min}$. Estas amostras foram utilizadas no cálculo

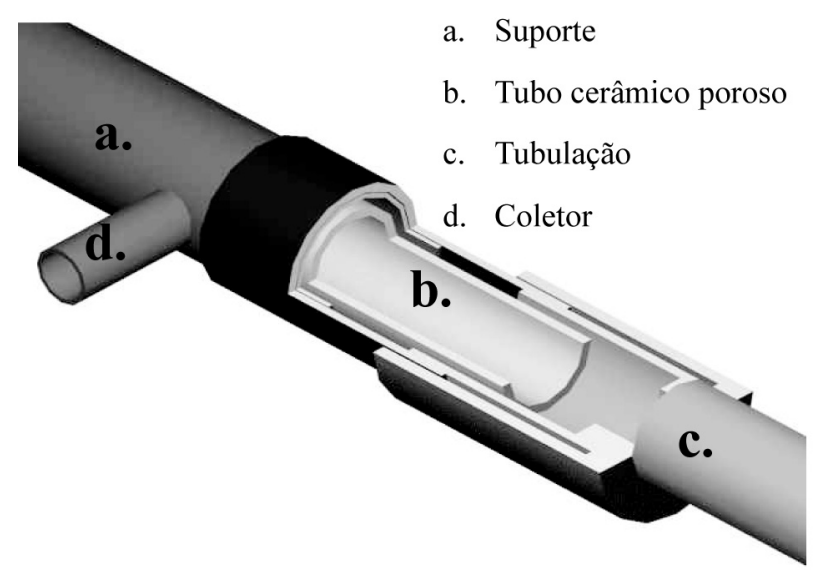

Figura 1: Detalhe da montagem do tubo cerâmico no equipamento de micro filtração.

[Figure 1: Ceramic tube assembling in the micro filtration equipment.] 
da vazão mássica, do teor de carbono, da condutividade elétrica e no cálculo do coeficiente de rejeição segundo a Equação B [2]:

$$
\mathrm{R}=100 \times\left(1-\frac{\mathrm{T}_{\mathrm{f}}}{\mathrm{T}_{\mathrm{e}}}\right)
$$

onde $\mathrm{R}$ é o coeficiente de rejeição percentual (\%), $\mathrm{T}_{\mathrm{f}} \mathrm{e}$ $\mathrm{T}_{\mathrm{e}}$ são os teores de carbono do filtrado e da emulsão, respectivamente.

A vazão mássica do filtrado, a pressão trans-membrana e as dimensões do tubo foram usadas para calcular o fluxo de filtrado segundo a Equação C [2]:

$$
\text { Fluxo }=\frac{V}{A \times t \times P}
$$

onde $\mathrm{V}$ é o volume de filtrado em litros, A é a área superficial do tubo $\mathrm{em} \mathrm{m}^{2}$, $\mathrm{t}$ o intervalo em horas entre as coletas de amostras de filtrado e $\mathrm{P}$ a pressão em bar.

Seguindo a metodologia proposta foi possível fazer uma avaliação da eficácia do material poroso obtido nesse trabalho, abrangendo todo o processo de fabricação do mesmo e seu desempenho em microfiltração de emulsões.

\section{RESULTADOS E DISCUSSÃO}

A Fig. 2 ilustra os resultados de porosidade das composições estudadas. Anterior à discussão destes resultados, cabe esclarecer a nomenclatura adotada para as composições avaliadas. O primeiro índice corresponde à fração em peso de sólidos (60\%-p e $80 \%$-p). Já o segundo, indica a temperatura de sinterização, onde 11 e 12 correspondem a $1150{ }^{\circ} \mathrm{C}$ e $1250{ }^{\circ} \mathrm{C}$, respectivamente. Finalmente, o terceiro índice refere-se ao tempo de patamar de queima: 1 ou $3 \mathrm{~h}$.

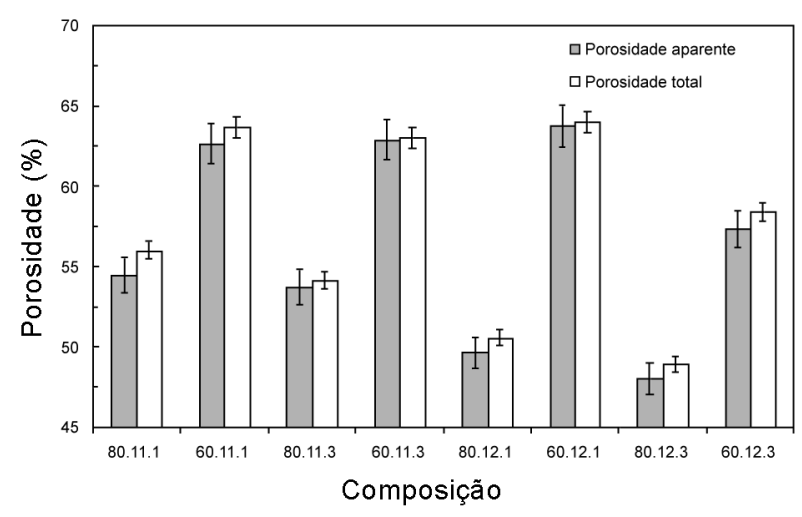

Figura 2: Porosidade aparente e total das composições estudadas. [Figure 2: Total and apparent porosity of all analyzed compositions.]
Os resultados apresentados na Fig. 2 revelam inicialmente que a porosidade total formada nas composições é em sua maior parte constituída de poros abertos (porosidade aparente). Isto é um dado importante, uma vez que a estrutura para microfiltração deve possuir alta porosidade associada a alta permeabilidade.

Quanto à fração de sólidos, vê-se que o aumento do teor de $60 \%$-p para $80 \%$-p reduziu significativamente a porosidade final da cerâmica. Esta redução da porosidade também foi observada quando do aumento da temperatura de sinterização e/ou tempo de patamar de queima.

Assim, considerando-se a faixa de porosidade recomendada na literatura [2] de $40 \%$ aos dados da Fig. 2 selecionaram-se as composições contendo 60\%-p de sólidos como as mais promissoras para produção dos tubos porosos.

Complementar à avaliação da porosidade, verificouse que a técnica de geração de poros pelo uso do aditivo inorgânico garantiu um acréscimo em torno de 20 pontos percentuais após queima, em relação à porosidade dos corpos verdes.

As amostras das composições estudadas foram também submetidas ao ensaio de permeabilidade ao ar em baixa temperatura (PBT), o qual permitiu o cálculo das constantes de permeabilidade $\mathrm{k}_{1}$ e $\mathrm{k}_{2}$ mostradas na Fig. 3 .

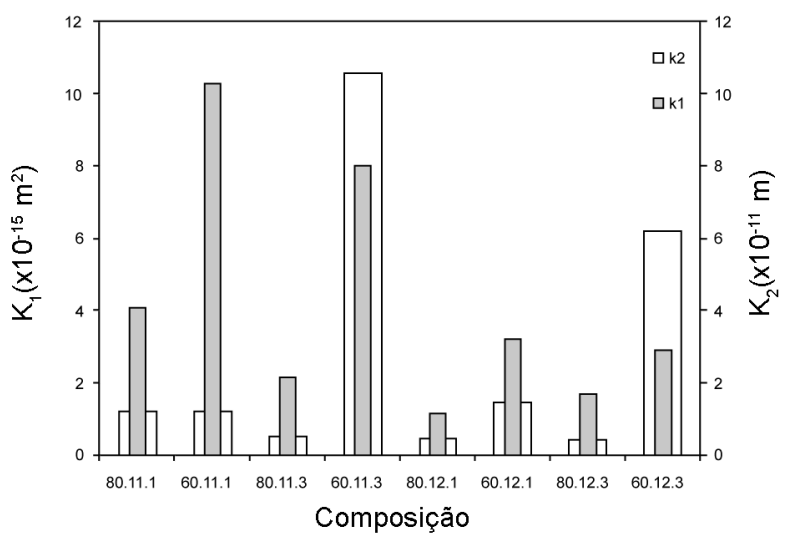

Figura 3: Constantes de permeabilidade Darciana $\left(\mathrm{k}_{1}\right)$ e não-Darciana $\left(\mathrm{k}_{2}\right)$ dos testes ao ar a baixa temperatura (PBT) para as composições porosas estudadas.

[Figure 3: Darcian $\left(k_{1}\right)$ and non-Darcian $\left(k_{2}\right)$ constants of permeability for tests with air at room temperature (LTP).]

Dentre as composições estudadas foi selecionada a composição 60.11.1 por apresentar o maior valor de $\mathrm{k}_{1} \mathrm{e}$ a composição 60.11 .3 , pelo seu maior valor de $\mathrm{k}_{2}$. Importante mencionar que os valores de permeabilidade aqui obtidos são maiores do que os apresentados na literatura $[2,4]$.

$\mathrm{O}$ termo $\mathrm{k}_{1}$ representa a interação entre o fluido e a superfície do material, enquanto $\mathrm{k}_{2}$ é um indicativo da tortuosidade da microestrutura do sistema.

Finalizando a caracterização física das oito composições avaliou-se através da porosimetria de mercúrio a distribuição 
do tamanho de poros nas mesmas. As curvas de volume intrudido de $\mathrm{Hg}$ para as composições estudadas encontramse na Fig. 4. Pode-se observar que a freqüência dos tamanhos dos poros presentes em todas as composições está distribuída em uma faixa estreita entre $0,1 \mu \mathrm{m}$ e $0,4 \mu \mathrm{m}$.

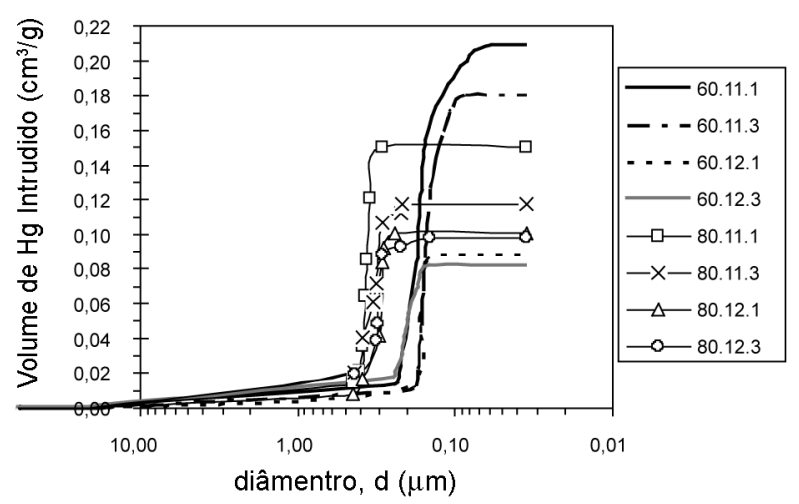

Figura 4: Curvas de volume de $\mathrm{Hg}$ intrudido para as composições estudadas.

[Figure 4: Curves for the intrusion of $\mathrm{Hg}$ for all analyzed compositions.]

Assim, considerando-se os resultados de porosidade, de permeabilidade e de distribuição de tamanho de poros, escolheu-se a composição 60.11 .3 como a mais adequada para a produção dos tubos cerâmicos para microfiltração.

A etapa seguinte consistiu em produzir estes tubos cerâmicos com comprimento de $25 \mathrm{~cm}$, diâmetro externo de $1 \mathrm{~cm}$, espessura de parede de $0,2 \mathrm{~cm}$, com a finalidade de submetê-los a ensaios de microfiltração tangencial.

O principal resultado obtido com o ensaio de microfiltração é o teor de carbono presente no filtrado, pois permite calcular o coeficiente de rejeição (Equação B) que é um indicativo da eficiência do filtro cerâmico produzido. $\mathrm{Na}$ Fig. 5 são apresentados os valores do coeficiente de rejeição em função da freqüência de operação da bomba e da pressão da emulsão de água com óleo de girassol. Na Fig. 6 esta

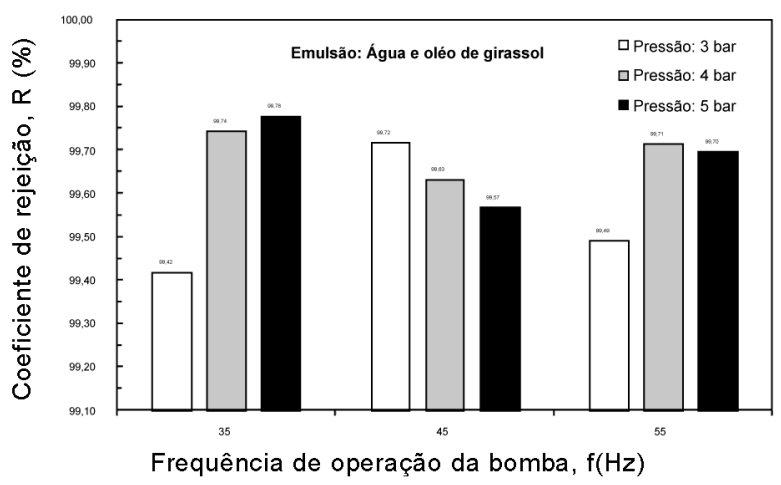

Figura 5: Coeficiente de rejeição para microfiltração de emulsão água/óleo.

[Figure 5: Rejection coefficient for microfiltration of oil-water emulsions.]

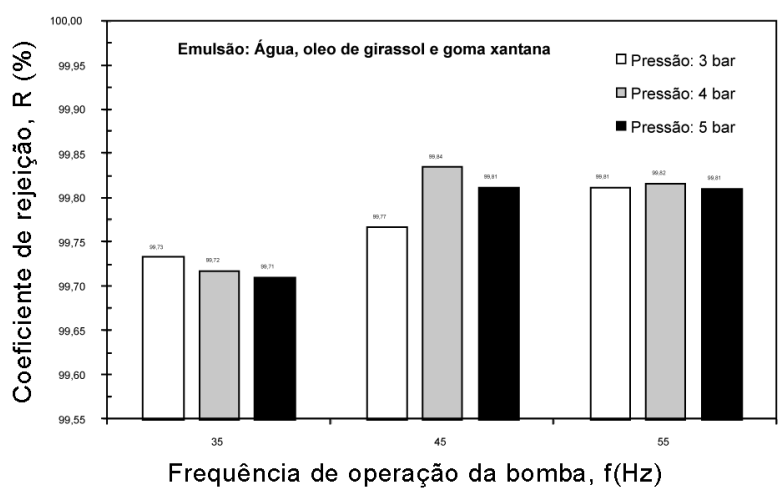

Figura 6: Coeficiente de rejeição para microfiltração de emulsão água/óleo/goma xantana.

[Figure 6: Rejection coefficient for microfiltration of oil-waterxantana gum emulsion.]

mesma avaliação é feita para emulsão de água com óleo de girassol e goma xantana.

É relevante o fato da eficiência de filtração (coeficiente de rejeição, R) dos tubos cerâmicos porosos ser sempre maior que $99,00 \%$, como sugerido pela literatura para tubos comerciais [2] e chegar ao máximo de 99,84\%. Este valor é suficiente para reduzir a concentração de carbono de $6548,00 \mathrm{mg} / \mathrm{L}$ para pouco mais de $10 \mathrm{mg} / \mathrm{L}$, enquanto que a água usada possuía $0,578 \mathrm{mg} / \mathrm{L}$.

Estes resultados comprovam que os tubos porosos desenvolvidos e produzidos em laboratório podem ser utilizados na microfiltração de emulsões, uma vez que a literatura recomenda coeficiente de rejeição maior do que $99,00 \%$ e fluxo entre 15 e $90\left(1 / \mathrm{m}^{2} \mathrm{~h}\right.$ bar $)$

Os valores de fluxo obtidos com o tubo cerâmico produzido ficaram entre 7 e $45\left(1 / \mathrm{m}^{2} \mathrm{~h}\right.$ bar $)$ e com valor médio de $27\left(1 / \mathrm{m}^{2} \mathrm{~h}\right.$ bar $)$.

\section{CONCLUSÕES}

Os resultados deste trabalho demonstraram a eficiência do aditivo inorgânico, associado ao processo de colagem de suspensões, na obtenção de tubos cerâmicos de elevada porosidade $(>60 \%)$, alta permeabilidade e com distribuição estreita de tamanho de poros pequenos $(0,1$ a $0,4 \mu \mathrm{m})$. Além disso, os testes de microfiltração de emulsões revelaram que os tubos cerâmicos porosos aqui produzidos apresentaram elevados valores de coeficiente de rejeição $(>99,4 \%)$ e fluxo médio de $27\left(1 / \mathrm{m}^{2} \mathrm{~h}\right.$ bar $)$, valores adequados para tubos cerâmicos comerciais. Desse modo, conclui-se que a técnica desenvolvida de obtenção dos tubos porosos pode ser utilizada na produção de cerâmicas porosas para aplicações em microfiltração de emulsões, tais como combustíveis, alimentos e cosméticos.

\section{AGRADECIMENTOS}

Ao Prof. Dr. Sérgio Rodrigues Fontes da EESC-USP pelos comentários e pela permissão do uso do equipamento 
de microfiltração, à Alcoa Alumínio S. A. e à Fapesp pelo apoio concedido na realização deste trabalho.

\section{REFERÊNCIAS}

[1] F. Q. Tang, H. Fudouzi, T. Uchikoshi, Y. Sakka, "Preparation of porous materials with controlled pore size and porosity", J. Eur. Ceram. Soc. 24, 2 (2004) 341-344.

[2] C. Yang, G. Zhang, N. Xu, J. Shi, "Preparation and application in oil-water separation of $\mathrm{ZrO}_{2} / \alpha-\mathrm{Al}_{2} \mathrm{O}_{3} \mathrm{MF}$ membrane", J. Membrane Sci. 142 (1998) 235-243.

[3] S. M. Joscelyne, G. Trägårdh, "Membrane emulsification -a literature review", J. Membrane Sci. 169 (2000) 107-117.

[4] P. M. Biesheuvel, H. Verwij, "Design of ceramic membrane supports: permeability, tensile strength and stress", J. Membrane Sci. 156 (1999) 141-152

[5] Y. Gu, G. Meng, "A model for ceramic membrane formation by dip-coating", J. Eur. Ceram. Soc. 19 (1999) 1961-1966.

[6] Z. Deng, J. Yang. Y. Beppu, M. Ando, T. Ohji, "Effect of agglomeration on mechanical properties of porous zirconia fabricated by partial sintering", J. Am. Ceram. Soc. 85, 9 (2002) 1961-1965.

(Rec. 21/06/2005, Ac. 02/01/2006) 